patients still get transient side-effects especially at the beginning of taking this regimen due to efavirenz as such, regular monitoring and thorough counselling of all patients on the side effects of tenofovir-based regimen and transient nature of side effects is needed. A large scale study to be done to obtain data on longterm side-effects of tenofovir-based regimen most possibly renal impairment due to tenofovir or efavirenz-induced gynecomastia.

\section{P17.26 VIRAL LOADS AMONG HIV-INFECTED PERSONS DIAGNOSED WITH PRIMARY AND SECONDARY SYPHILIS IN FOUR US CITIES: NEW YORK CITY, PHILADELPHIA, PA, WASHINGTON, DC, AND PHOENIX, AZ}

\begin{abstract}
1,2,3 Melanie M Taylor*, 'Daniel R Newman, ${ }^{1,4}$ Julia A Schillinger, ${ }^{1,5}$ Felicia MT Lewis, ${ }^{1,6}$ Bruce Furness, ${ }^{4}$ Sarah Braunstein, ${ }^{4}$ Tom Mickey, ${ }^{2}$ Julia Skinner, ${ }^{5}$ Michael Eberhart, ${ }^{6}$ Jenevieve Opoku, ${ }^{1,4}$ Susan Blank, ${ }^{1}$ Thomas A Peterman. ${ }^{1}$ Centers for Disease Control and Prevention, Division of STD Prevention, Atlanta, GA, USA; ${ }^{2}$ Arizona Department of Health Services, STD Program, Phoenix, AZ, USA; ${ }^{3}$ Maricopa County Department of Public Health, STD Program, Phoenix, AZ, USA; ${ }^{4}$ New York City Department of Health and Mental Hygiene, Long Island City, NY, USA; ${ }^{5}$ Philadelphia Department of Public Health, Philadelphia, PA, USA; ${ }^{6}$ HIVIAIDS, Hepatitis, STD and TB Administration, District of Columbia Department of Health, Washington DC, USA
\end{abstract}

\subsection{6/sextrans-2015-052270.604}

The findings and conclusions in this report are those of the authors and do not necessarily represent views of the Centres for Disease Control and Prevention.

Background Incident syphilis among HIV-infected persons indicates ongoing behavioural risk for HIV transmission. Detectable viral loads among co-infected cases may amplify this risk.

Methods Primary and secondary (P\&S) cases reported during 2009-2010 from four US sites were cross-matched to local HIV surveillance registries to identify syphilis case-persons infected with HIV prior to or shortly after the syphilis diagnosis. We examined HIV viral load and CD4 results collected within 6 months before or after syphilis diagnosis for the co-infected cases identified. Independent correlates of detectable viral loads ( $\geq 200$ copies $/ \mathrm{mL}$ ) were determined.

Results We identified 1675 cases of incident primary or secondary syphilis among persons with HIV. Median age was 37 years, 99.5\% were male, 41.1\% were African American, 24.5\% Hispanic, and $79.9 \%$ of the HIV diagnoses were made at least one year prior to syphilis diagnosis. Among those co-infected, there were no viral load results reported for 188 (11.2\%); of the 1487 (88.8\%) with reported viral load results, 809 (54.4\%) had a detectable viral load (median 25,101 copies/mL, range 2063,590,000 copies $/ \mathrm{mL}$ ). Detectable viral loads were independently correlated with syphilis diagnosed at younger age, at an STD clinic, and closer in time to HIV diagnosis.

Conclusion More than half of syphilis case-persons identified with HIV had a detectable viral load collected within 6 months of the syphilis diagnosis. This suggests virologic as well as active behavioural risk for transmitting HIV.

\section{P17.27 DECENTRALISING HIV VIRAL LOAD TESTING TO A REGIONAL LABORATORY IN AGADIR, SOUTHERN MOROCCO}

${ }^{1}$ Houda Eloudyi ${ }^{*},{ }^{1}$ Sanae Lemrabet, ${ }^{2}$ Mohamed Aghrouch, ${ }^{2}$ Samira Kharbouch, ${ }^{1}$ Hicham Oumzil. ${ }^{1}$ National Referral Laboratory of HIV, National Institute of Health, Rabat, Morocco; '2aboratoryOf Biology, Hassan II Hospital, Agadir, Morocco

10.1136/sextrans-2015-052270.605
Despite the increase of HIV patient throughput in Morocco, follow up testing (HIV Viral load VL) is still centralised in the National referral laboratory (NRL) as the activity required trained staff and specialised infrastructure.

Patients were often lost due to great distances between testing centre and home as well as delays in returning results.

To follow the dynamic of decentralising HIV treatment and care in Morocco, the NRLH launched a process of strengthening regional laboratory capacities.

In this framework, we assessed factors associated with lab capacities to offer HIV viral load testing, and followed their performance after implementation.

On site visits were performed to the laboratory of the regional laboratory of the Hassan II hospital of Agadir in southern Morocco; this region that accounts the greatest number of HIV positive cases. Using Laboratory assessment tool, the laboratory capacities were evaluated, and gaps related to the facility layout, human resources, training, equipment and reagent were fixed thank to the Global Fund for HIV support.

A follow up the lab performance was set for a period of two months, by retesting all the samples $(n=194)$ at the NRL. Data were analysed using MedCalc software to calculate the Spearman's coefficient of rank correlation (rho).

VL results were ranged from 150 to 221242 copies $/ \mathrm{ml}$.

A perfect match of VL results between the measurements at the NRL and at the Agadir regional lab was observed. The observed Spearman correlation index was of 0.98. P $<0,0001$ (95\% CI: 0,973 to 0,990 ).

In the light of these results, HIV VL testing was moved to the periphery and closer to the site of therapeutic management structures. Patients could get their results conveniently and quickly. This experience is worth to reproduce in other regions of the kingdom were HIV prevalence is steadily increasing.

\section{P17.28 ASSESSMENT OF HIV-1 PRIMARY DRUG RESISTANCE MUTATIONS IN ANTIRETROVIRAL THERAPY-NAIVE CASES IN MOROCCO}

${ }^{1} \mathrm{H}$ Eloudyi*, 'S Lemrabet, ${ }^{2} \mathrm{M}$ Malmoussi, ${ }^{2} \mathrm{Z}$ Ouagari, ${ }^{1} \mathrm{E}$ Elharti, ${ }^{1} \mathrm{M}$ Akrim, ${ }^{1} \mathrm{H}$ Oumzil. ${ }^{1}$ Laboratoire National de Référence Pour Le VIH. Institut National d'Hygiène, Rabat; ${ }^{2}$ Centre Référent VIH, Hôpital Hassan II, Agadir

\subsection{6/sextrans-2015-052270.606}

Antiretroviral drug resistance is a major challenge for management and control of HIV-1 infection worldwide and particularly in resource limited countries.

Although combined antiretroviral drug therapy has greatly improved the life-span and the life quality of the patients, HIV-1 drug resistance poses a major obstacle for treatment outcome.

This study aims to determine the prevalence of primary resistance in a group of newly diagnosed patients naïve to treatment, in the region of Souss Massa Draa in sounthern Morocco.

A total of 47 antiretroviral treatment (ART) naïve patients were included. Virological status was determined by Real time PCR (Abbott, USA).

Primary drug resistance mutations were identified according to the Stanford HIV database and the algorithm of the National Agency for AIDS Research (ANRS). The clinical staging of patients was made upon the International Classification of the Centres for Disease Control (CDC).

Ninetheen $19 \%$ of recruits were diagnosed in stage A, $11 \%$ in stage $\mathrm{B}$, and the large proportion of patients in stage C $38 \%$, while $32 \%$ of which the stadium has not been determined. 